\title{
Une crise sanitaire avant tout
}

\author{
Above All, a Health Crisis
}

\section{F. Braun}

Reçu le 28 juillet 2020 ; accepté le 18 août 2020

(C) SFMU et Lavoisier SAS 2020

Notre pays fait face à une crise sanitaire d'une ampleur sans précédent depuis des décennies. Même si cette crise n'est pas terminée et que nos retours d'expérience sont encore en cours, il est essentiel de partager au plus vite les conclusions que nous pouvons déjà en tirer : c'est l'objectif des articles qui suivent. Nos réponses, les réponses du système de santé, ont suivi la cinétique de cette crise en anticipant et en s'y adaptant sans cesse, tenant compte des connaissances sur ce virus et cette pandémie qui ne cessent de progresser. C'est sur la base de cette cinétique qu'il convient d'analyser nos actions.

Début janvier 2020, lorsque nous étions informés de la pandémie en Chine, le risque paraissait lointain pour tous. La réunion, le 4 février 2020, de l'ensemble des responsables de Samu-Centre 15 nous permettait d'échanger sur l'évolution de nos plateformes de régulation médicale vers le service d'accès aux soins (SAS) : c'était prémonitoire [1]. Fin février 2020, le cluster de Creil nous a confirmé que cette crise sanitaire risquait de s'étendre rapidement et que les Samu-Centre 15 étaient en première ligne : de 400 dossiers de régulation médicale traités le vendredi, le SamuCentre 15 de l'Oise passait à 1400 dossiers le dimanche... Les Samu et les services d'urgence des Hauts-de-France se sont organisés avec le renfort du Samu de Paris, Samu zonal d'Île-de-France, pour répondre à cette surcharge. Nous échangions quotidiennement entre nous, les bonnes idées diffusaient rapidement. Mi-mars 2020 (entre le 10 et le 15 mars), les Samu-Centre 15 sur tout le territoire national ont fait face à une multiplication par 3, voire 4 de leurs activités, augmentation brutale (en 24 heures). Les courbes des appels aux numéros d'urgence (Fig. 1) témoignent que seul le « 15 » a subi cette vague imprévisible. Nous avons alors adapté nos effectifs, nos moyens techniques et nos organisations pour y faire face [2]. Cette augmentation d'appels ne s'est pas alors traduite, sur le terrain, par un engagement de moyens de réanimation (Smur) ou de transports sanitaires

\section{F. Braun $(\bowtie)$}

Samu et Urgences de France,

103, boulevard Magenta, F-75010 Paris, France

e-mail : president@sudf.fr supplémentaires, mais par une augmentation des parcours de soins non hospitaliers. Le 15 mars 2020, nos collègues de Mulhouse et de Colmar nous alertaient sur la situation dans le Haut-Rhin qui avait quelques jours d'avance sur le Bas-Rhin et la Moselle et une semaine sur l'Île-de-France. "Depuis trois jours, nous sommes submergés aux urgences par un flux incessant de patients avec critères d'hospitalisation, AEG, hypoxémie importante, pneumopathies bilatérales... le taux d'hospitalisation après passage aux urgences est de $40 \%$. " "Les lits de réanimation de la région sont saturés, et impossible de trouver des respirateurs pour ouvrir de nouveaux postes de réa. » «Durant ces 15 derniers jours, toutes les mesures que nous avons prises ont été dépassées et donc insuffisantes dans la journée même, tant la cinétique est rapide. " "Préparez-vous, ainsi que vos personnels, à cette vague majeure. Il y avait un avantCovid-19, il y aura un après-Covid-19 avec de très lourdes cicatrices $»^{1}[3]$. Cette information, largement diffusée, permettra à tous de se préparer activement. Une semaine plus tard, les patients graves se multipliaient : les services de réanimation des zones les plus touchées se remplissaient à toute vitesse. La simple « grippette » annoncée par certains se révélait, en France comme en Italie quelques jours plus tôt, pourvoyeuse de cas graves et mortels. Nos Smur et les services d'urgence étaient alors fortement sollicités, et la charge psychologique terrible pour les soignants. Après la prise en charge téléphonique et « en ville » de patients peu graves, nous étions confrontés à une vague de patients graves dont nous identifierons, malheureusement après, qu'elle était prévisible [4].

S'est ouverte alors pour nous une deuxième phase avec la saturation des capacités de réanimation dans les régions les plus touchées (Grand-Est et Île-de-France) et la mise en œuvre de transferts massifs de patients de réanimation. Ces transferts interrégionaux et internationaux ont permis d'éviter le débordement des capacités de réanimation et de sauver

\footnotetext{
${ }^{1}$ Courriel des Drs Marc Noizet (Mulhouse) et Yannick Gottwalles (Colmar) du 15 mars 2020 adressé au président de Samu-Urgences de France.
} 


\section{APPELS D'URGENCE TOUS OPÉRATEURS (MÉTROPOLE)}

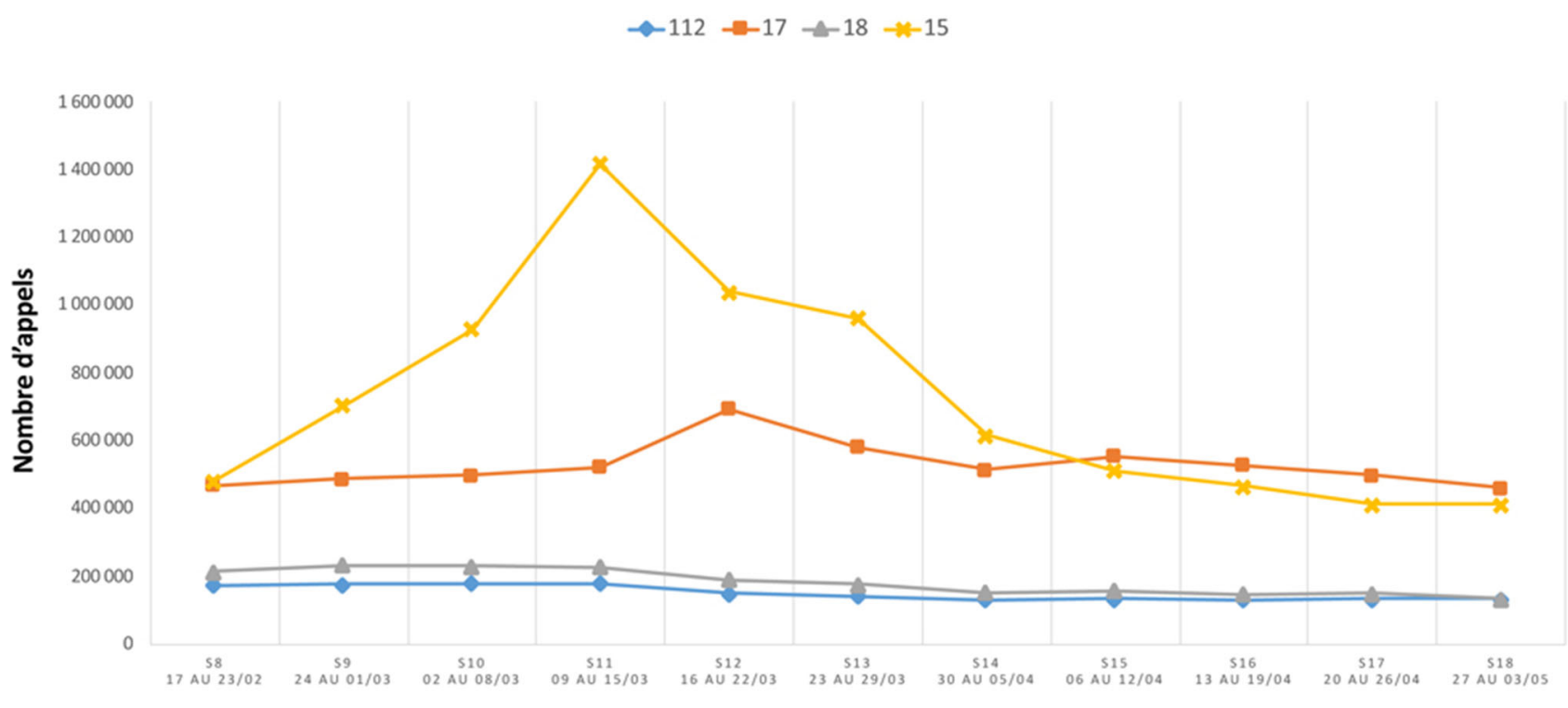

Période

Fig. 1 Nombre d'appels hebdomadaires passés vers les principaux numéros d'urgence (tous opérateurs de téléphonie) en France métropolitaine du 17 février au 3 mai 2020 pendant la crise Covd-19. 15=Samu, 17=police/gendarmerie, 18= sapeurs-pompiers, 112 = sapeurs-pompiers (80 \%) ou Samu (20\%) [source : ministère de la Santé, Direction générale de l'offre de soins (DGOS) : groupe de travail « Plateforme de localisation des appels d'urgence »]

des vies. Pendant ce temps, nous avons constaté une chute drastique de la fréquentation des services d'urgence $(-50 \%)$ constat d'un "refus aux soins" de patients inquiets de contracter le virus à l'hôpital : ils seront pris en charge à la fin du confinement avec des conditions médicales parfois dégradées. Il nous a fallu ensuite, et jusqu'à aujourd'hui, assurer le suivi et le rapatriement des patients transférés et participer au « redémarrage » de l'hôpital avec la volonté de ne pas revenir à « l'anormalité d'avant»!

Dans cette crise sanitaire, l'action des services d'urgence et des Samu-Centre 15 a été prépondérante. À la phase initiale, pour apporter une réponse médicale adaptée à toutes les sollicitations de nos concitoyens qui étaient inquiets d'être malades du coronavirus ou qui, victimes ou non du virus, présentaient des signes de gravité, et dans un second temps en évitant, par des transferts, la saturation des services de réanimation. Proposer des parcours de soins adaptés est l'objectif fondamental de la régulation médicale. Dans cette crise sanitaire, il s'est doublé de celui d'éviter impérativement la saturation des services d'urgence, risque majeur de désorganisation du système de santé et de contaminations supplémentaires. Nous pouvons évaluer à 80000 par jour le nombre d'appels supplémentaires au moment du pic : que penser si ces 80000 patients, faute de régulation médicale, s'étaient ou avaient été dirigés vers les services d'urgences ? L'action de la régulation médicale, en synergie avec nos collègues généralistes du premier recours et les établissements publics comme privés, a permis d'éviter cet écueil. Au cœur de la crise ensuite, il était essentiel d'éviter le débordement des capacités des soins critiques pour leurs permettre de continuer à prendre en charge les patients qui en avaient besoin était essentiel, et les Samu-Centre 15 ont dû gérer des transferts en nombre de patients de réanimation. Ces transferts ont libéré 644 lits de réanimation en Grand-Est et en Îlede-France [5-9].

Pour nous, professionnels de santé, tirer les enseignements d'une crise n'est pas uniquement analyser comment la crise a été gérée en se demandant ce qu'il aurait fallu faire pour la gérer autrement, mais comment, en fonction de ce qui s'est passé, améliorer nos capacités à affronter la crise future qui, par définition, ne ressemblera pas à celle-ci. Comme au plus fort de la crise, loin de chercher des responsabilités, nous recherchons des solutions.

Nous avons beaucoup entendu, et dit, que cette crise était inédite, mais en quoi ? Malgré une couverture médiatique « hors normes », cette crise sanitaire est restée virtuelle pour beaucoup de nos concitoyens : il fallait vraiment être directement confronté aux malades graves, ceux de réanimation, 
pour en comprendre l'ampleur. Le rapport fait par le Pr Benoit Vallet à l'Assistance publique-Hôpitaux de Paris après une mission d'évaluation à Mulhouse en est un bon exemple [10]. Cette « maladie » a imposé un cadrage contradictoire dont nous n'avons pas l'habitude : à la fois maladie grave et bénigne, ni une « grippette » ni « Ebola », cette dualité a imposé une gestion coordonnée des malades pour laquelle la régulation médicale a joué le rôle indispensable de chef d'orchestre. Cette dualité « grave-pas grave » explique que ce n'est pas par des visions catégorielles, en silos, que nous devons tirer les enseignements de cette pandémie, mais bien en analysant toutes ses composantes sanitaires.

Au-delà des structures de médecine d'urgence, cette crise sanitaire a été un véritable « stress-test » pour notre système de santé qui nous a prouvé que lorsque « soignants » et « administratifs » travaillent avec le même objectif, font preuve d'agilité, d'adaptabilité, nous pouvons tous donner le meilleur de nous-mêmes.

Liens d'intérêts : l'auteur déclare ne pas avoir de liens d'intérêts.

\section{Références}

1. Mesnier T, Carli P (2019) Rapport : Pour un pacte de refondation des urgences. https: solidarités-sante.gouv.fr/IMG/pdf/rapport pour_un_pacte_de_refondation_des_urgences_2019-058r.pdf (Dernier accès le 4 août 2020)
2. Telion C, Marx J-S, Dautreppe C, Carli P (2020) Retour d'expérience sur la régulation au Samu de Paris pendant la crise Covid19. Ann Fr Med Urgences 10:202-11

3. Noizet M, Gottwalles Y, Nguyen S, et al (2020) Retour d'expérience de deux structures d'urgences de centres hospitaliers généraux pendant la crise Covid-19. Ann Fr Med Urgence 10: 243-50

4. Piarroux R, Batteux F, Rebaudet S, Boelle PY (2020) Les indicateurs d'alerte et de surveillance de la Covid-19. Ann Fr Med Urgence 10:333-9

5. Chollet-Xémard C, Michel D, Szuster P, et al (2020) Retour d'expérience des transferts en HéliSmur de patients Covid-19. Ann Fr Med Urgences 10:266-71

6. Beaussac M, Distinguin B, Turc J, Boutonnet M (2020) Retour d'expérience des six évacuations sanitaires aériennes collectives MoRPHEE durant la pandémie Covid-19. Ann Fr Med Urgences 10:272-7

7. Habchi K, Oulehri N, Noizet M, et al (2020) Covid-19 : les transferts internationaux de patients de réanimation, une solution pour les départements frontaliers. Ann Fr Med Urgences 10:298-305

8. Lamhaut L, Nivet CM, Dagron C, et al (2020) Retour d'expérience des évacuations par train à grande vitesse de patients en syndrome de détresse respiratoire aiguë sur infection à Covid19 : les missions Chardon. Ann Fr Med Urgences 10:288-97

9. Lecarpentier E, Travers N, Jbeili C, Chollet-Xémard C (2020) Retour d'expérience sur le centre médical de coordination et d'évacuation aérien Covid - Paris-Orly. Ann Fr Med Urgences 10:278-87

10. Vallet B (2020) Le Grand-Est face à la crise sanitaire : le $\mathrm{Pr}$ Benoît Vallet, ex-DGS, témoigne. Rev Prat Newletter. https:// www.larevuedupraticien.fr/article/le-grand-est-face-la-crise-sanitaire-le-pr-benoit-vallet-ex-dgs-temoigne (Dernier accès le 8 août 2020) 